\title{
Editorial
}

\section{CD-ROMance or tragedy}

In 1984-1985, the information world began to be assaulted by multiple inputs relating to this technology. While video disks had been creeping into our environment in a relatively peaceful and unobtrusive fashion, the compact disks have virtually leaped upon us.

We who have made the distribution transitions based on microforms, online, and most recently downloading, during three decades, have a strong sense of déjà vu as meeting after meeting, article after article and the entire array of communications available in our industry have focused on this medium.

Recently, H.E. Kennedy, president of BIOSIS, delivered the Miles Conrad Lecture at the annual meeting of the National Federation of Abstracting and Information Services (NFAIS). In that lecture Kennedy wondered whether the information industry was driven by available technology, as opposed to using the technological opportunities to fill real needs. He suspected the former to be the case, and CD-ROM may be the latest example of that fallacy.

Dr. Kennedy and your editor are not alone in this sceptical position. Barbara Quint, editor-in-chief of the very useful magazine Database End-user, in the March 1986 issue of that publication, questions the claims for cheap searching, unlimited use, full online access and fixed predictable costs offered by the proponents of the disks, and points out that motivations of the end-users and those of the data-base producers may be irreconcilable.

CD-ROMs will find their niche and may well turn out to be important factors for information delivery-but let them achieve their role as a result of their utility and not as the latest meeting topic fad.

A.W. Elias 